\title{
A importância da microbiota intestinal e seus efeitos na obesidade
}

\author{
The importance of the intestinal microbiota and its effects on obesity \\ La importância de la microbiota intestinal y sus efectos sobre la obesidad
}

Recebido: 14/05/2021 | Revisado: 20/05/2021 | Aceito: 22/05/2021 | Publicado: 08/06/2021

\author{
Cecília Santa Cruz de Souza \\ ORCID: https://orcid.org/0000-0002-7462-7637 \\ Centro Universitário Fametro, Brasil \\ E-mail: ceciliasouzanutri@gmail.com \\ Rebecca Carvalho de Souza \\ ORCID: https://orcid.org/0000-0003-0152-3412 \\ Centro Universitário Fametro, Brasil \\ E-mail: rebeccaalbuq@icloud.com \\ Juliana do Nascimento Evangelista \\ ORCID: https://orcid.org/0000-0001-6116-6555 \\ Centro Universitário Fametro, Brasil \\ E-mail: julianaevangel@hotmail.com \\ José Carlos de Sales Ferreira \\ ORCID: https://orcid.org/0000-0002-1867-8229 \\ Centro Universitário Fametro, Brasil \\ E-mail: jcarlos.sales@gmail.com
}

\begin{abstract}
Resumo
Introdução: A microbiota intestinal humana possui um papel fundamental na manutenção da saúde e prevenção de doenças como por exemplo a Obesidade. Sabe-se que há correlação entre ambas, visto que esta condição clínica acarreta a Disbiose Intestinal. E, a terapêutica envolve também a modulação intestinal com prebióticos e probióticos. Objetivo: Descrever a importância do equilíbrio da microbiota intestinal na prevenção e tratamento da obesidade. Metodologia: Trata-se de uma revisão bibliográfica com abordagem descritiva. Resultados: A partir do estudo de literatura foi possível observar que além da influência de fatores anatômicos e fisiológicos, existem causas multifatoriais que levam o indivíduo a obesidade, como o desequilíbrio da microbiota intestinal, bem como hábitos alimentares ruins. Discussão dos resultados: Apresentou-se a existência efetiva de uma correlação entre a Microbiota Intestinal e a Obesidade ocasionada pelo tipo de alimentação do indivíduo, uso indevido de fármacos, alimentos ricos em FODMAPs e que se pode tratar com mudanças no estilo de vida e modulação intestinal. Conclusão: Através dos estudos realizados foi possível comprovar a importância de manter o equilíbrio microbiano intestinal a fim de evitar complicações clínicas e melhorar o prognóstico do indivíduo obeso.
\end{abstract}

Palavras-chave: Microbiota intestinal; Obesidade; Disbiose; Probióticos; Prebióticos.

\begin{abstract}
Introduction: The human intestinal microbiota has a fundamental role in maintaining health and preventing diseases such as obesity. It is known that there is a correlation between both, since this clinical condition leads to Intestinal Dysbiosis. And, the therapy also involves an intestinal modulation with prebiotics and probiotics. Objective: To describe the importance of the balance of the intestinal microbiota in the prevention and treatment of obesity. Methodology: This is a bibliographic review with a descriptive approach. Results: From the literature study, it was possible to observe that in addition to the influence of anatomical and physiological factors, there are multifactorial causes that lead the individual to obesity, such as the imbalance of the intestinal microbiota, as well as bad eating habits. Discussion of the results: The effective existence of a correlation between the Intestinal Microbiota and Obesity caused by the type of diet of the individual, medicine misuse, foods rich in FODMAPs and which can be dealt with changes in lifestyle and intestinal modulation. Conclusion: Through the studies carried out, it was possible to prove the importance of maintaining the intestinal microbial balance in order to avoid clinical complications and improve the prognosis of the obese individual.
\end{abstract}

Keywords: Intestinal microbiota; Obesity; Dysbiosis; Probiotic; Prebiotic.

\section{Resumen}

Introducción: La microbiota intestinal humana tiene un papel fundamental en el mantenimiento de la salud y la prevención de enfermedades como la obesidad. Se sabe que existe una correlación entre ambos, ya que esta condición clínica conduce a la Disbiosis Intestinal. Y la terapia también implica la modulación intestinal con prebióticos y probióticos. Objetivo: Describir la importancia del equilibrio de la microbiota intestinal en la prevención y tratamiento de la obesidad. Metodología: Se trata de una revisión bibliográfica con enfoque descriptivo. Resultados: Del estudio 
de la literatura se pudo observar que además de la influencia de factores anatómicos y fisiológicos, existen causas multifactoriales que llevan al individuo a la obesidad, como el desequilibrio de la microbiota intestinal, así como los malos hábitos alimenticios. Discusión de los resultados: Se presentó la existencia efectiva de una correlación entre la Microbiota Intestinal y la Obesidad causada por el tipo de dieta del individuo, abuso de fármacos, alimentos ricos en FODMAP y que puedan ser tratados con cambios en el estilo de vida y modulación intestinal. Conclusión: A través de los estudios realizados, se pudo comprobar la importancia de mantener el equilibrio microbiano intestinal para evitar complicaciones clínicas y mejorar el pronóstico del individuo obeso.

Palabras clave: Microbiota intestinal; Obesidad; Disbiosis; Probióticos; Prebióticos.

\section{Introdução}

A microbiota intestinal é composta por aproximadamente 100 trilhões de bactérias e exerce importante função no organismo humano (Silva, Santos \& Bressan, 2013). Estudos estão sendo realizados como alternativas no tratamento da Obesidade, visto que há evidências sobre indivíduos eutróficos e obesos possuírem a composição da microbiota intestinal distinta (Million et al., 2013; Stenman et al., 2015). Assim, dois filos estão sendo analisados como Firmicutes e Bacteroidetes e encontram-se no Intestino Delgado e Grosso.

Acrescido disso, a microbiota intestinal humana tem seu início de desenvolvimento na hora do parto gestacional e logo após com a amamentação ou introdução de leites artificiais precocemente, fazendo com que comecem a surgir comunidades microbianas (Bervoets et al., 2013). Além disso, uma menor diversidade de microrganismos é característica de indivíduos obesos, por exemplo (Silva, Santos \& Bressan, 2013). Outrossim, as relações que a microbiota intestinal exerce sobre os diversos sistemas do organismo refletem também na manutenção do metabolismo. A microbiota afeta tanto a aquisição de nutrientes quanto a regulação da energia adquirida (Bervoets et al., 2013).

Essa desregulação da microbiota intestinal tem relevância no desenvolvimento ou agravamento da obesidade, já que a enfermidade condiz com desordens metabólicas e inflamatórias que afetam e alteram os sistemas do organismo como um todo (Silva, Santos \& Bressan, 2013). Tendo em vista o eixo cérebro-intestino como uma legítima interligação em suas funções, a Obesidade influencia negativamente para o aumento de colonizações bacterianas patogênicas, contribuindo para o surgimento de outras doenças (Jumpertz et al., 2011).

Ademais, a Obesidade é um dos maiores problemas de saúde pública e além de ser uma patologia crônica possui causas multifatoriais como genética, psicossociais, psicoemocionais, endócrinas e hábitos diários (Park, Seo \& Youn, 2013). Assim, é caracterizada pelo elevado índice de massa corporal (IMC) constituindo uma síndrome metabólica de causa multifatorial que está relacionada a mecanismos biológicos, ambientais e neuropsicológicos (Bernhard et al., 2013).

Assim como também, pesquisas relacionaram os simbióticos, ação conjunta de prebióticos e probióticos, como grandes beneficiadores da saúde intestinal, podendo ainda auxiliar no tratamento e prevenção da doença (Rodrigues, 2011). E segundo Hill et al. (2014) caracteriza probióticos como sendo microrganismos vivos que quando administrados em doses adequadas, conferem benefícios a saúde. Dentre os efeitos benéficos temos: Normalização da microbiota, diminuição da permeabilidade intestinal, proteção contra invasores patogênicos e estimulação do sistema imunológico (Santos \& Varavalho, 2011).

Por conseguinte, estima-se que a modulação da microbiota intestinal seja o caminho para o tratamento de indivíduos classificados como obesos, como por exemplo o uso terapêutico dos probióticos (FAO WHO, 2001). Mas também, a desordem causada pelo excesso de microrganismos patogênicos, gerando uma alta permeabilidade no intestino chama-se Disbiose Intestinal (Santos \& Ricci, 2016). Acredita-se que pessoas com obesidade também a possuam.

Além do mais, a dieta ocidental caracterizada pelo alto consumo de gorduras e baixo em fibras, é um dos principais fatores que contribuem para a proliferação de bactérias patogênicas na microbiota intestinal (Jumpertz et al., 2011). A 
manutenção da saúde intestinal além de ser uma terapia nutricional para a doença crônica não transmissível, Obesidade, é garantia de bem-estar, longevidade e prevenção contra demais patologias.

Dessa forma, o presente artigo objetivou-se descrever a importância do equilíbrio da microbiota intestinal na prevenção da obesidade.

\section{Metodologia}

O presente estudo efetivou-se através de pesquisa descritiva, na qual propôs a identificação, registro e análise das características, fatores ou variáveis que se relacionam com o fenômeno ou processo (Perovano, 2014). Dessa forma, embasada em referências bibliográficas e científicas, relatando a importância da microbiota intestinal e seus efeitos a obesidade.

Para elaboração da pesquisa, foram realizados levantamentos bibliográficos fazendo o uso de materiais publicados em forma de revistas, órgãos oficiais, artigos em sites como SCIELO (Scientific Eletronic Library), PubMed (Serviço da National Library of Medicine) e LILACS. Para a busca dos artigos, utilizaram-se os descritores: Microbiota Intestinal; Obesidade; Disbiose; Probiótico; Prebiótico.

Dentre as referências buscadas, no caráter de inclusão, foram utilizadas referências entre 2010 e 2020 sobre o tema a ser detalhado e foram excluídos os quais não haviam compatibilidade com o assunto proposto, bem como os que não apresentavam informações completas de uso para elaboração do projeto, como por exemplo Doenças Inflamatórias Intestinais (DII). Além disso, analisaram minuciosamente a leitura de cada artigo na intenção de extrair todas as informações necessárias para a realização do conteúdo do projeto, a fim de se obter resultados satisfatórios.

\section{Resultados e Discussão}

\subsection{Microbiota Intestinal e Obesidade}

\subsubsection{Microbiota Intestinal}

A microbiota intestinal é composta principalmente por 5 filos e povoa principalmente o intestino grosso (Murphy et al., 2010). Ainda assim, a microbiota intestinal humana auxilia e contribui para o metabolismo de forma geral, exercendo importante função em converter o alimento em nutrientes e energia (Silva, Santos \& Bressan, 2013). Ou seja, ela é primordial para manutenção e qualidade de vida do indivíduo.

Existem dois filos que se encontram em maior proporção no intestino, são eles: Firmicutes e Bacteroidetes (Tremaroli \& Backhed, 2012). Bactérias Firmicutes são encontradas em maior quantidade no organismo adulto obeso e realizam a fermentação de carboidratos insolúveis, modificação de carboidratos complexos em glicose e ácidos graxos de cadeia curta, ou seja, podem aumentar o acúmulo de gordura (Valero, Colina \& Herrara, 2015).

Assim como também, os Bacteroidetes são encontrados em menor quantidade e utilizam os carboidratos de forma mais simples, a partir da metabolização de carboidratos insolúveis (Valero, Colina \& Herrara, 2015). Logo, percebe-se que há uma diferença na composição da microbiota intestinal entre obesos e eutróficos. Além disso, a composição da microbiota intestinal pode ser alterada a partir da alimentação, da idade e da genética (Wu et al., 2011).

De acordo com Barbosa et al. (2010) o principal mecanismo desempenhado pela microbiota bacteriana é a resistência à colonização. Mais comum no lúmen e nas superfícies da mucosa pela produção de componentes metabólicos tóxicos, como ácidos graxos de cadeia curta e de substâncias antimicrobianas como bacteriocinas. Além do mais, deve-se ressaltar que a resistência a colonização ocorre também por outros fatores como anatômicos e fisiológicos (incluindo a integridade da mucosa), salivação, secreção da Imunoglobulina IgA, produção de ácidos graxos, descamação da mucosa e motilidade gastrointestinal. 
Ademais, a constituição da microbiota intestinal é afetada por muitas variáveis desde a infância, incluindo o tipo de dieta (amamentação ou alimentação artificial), tipo de parto (vaginal ou cesariana), ambiente, fatores culturais e ambiente familiar (Matamoros et al., 2013). Ou seja, o microbioma é formado desde o parto até a vida adulta. Bello et al. (2016), relata que crianças nascidas de parto vaginal (PV) abrigam uma comunidade de bactérias que se assemelham às da vagina materna, em contrapartida, bebes que nascem de parto cesáreo (PC) apresentam uma flora bacteriana diferente. Portanto, há mais de 10 anos iniciou-se uma preocupação em estudar a relação direta entre a microbiota intestinal e obesidade já que há comprovações de que a modulação intestinal deve-se iniciar desde a infância para evitar patologias futuras.

\subsubsection{Obesidade}

A obesidade é considerada uma Doença Crônica Não Transmissíveis (DCNT) e um dos maiores problemas de saúde pública. É caracterizada por um acúmulo excessivo de tecido adiposo e consequências ruinosa a saúde (Oliveira et al., 2017). E é causada por um excesso no consumo de calorias na alimentação, sendo superior a energia que é usada pelo organismo para sua manutenção e realização das atividades diárias.

No Brasil, segundo a Pesquisa de Vigilância de Fatores de Risco e Proteção para doenças Crônicas por Inquérito Telefônico (VIGITEL) de 2018, o Brasil atingiu o maior índice de obesidade. A prevalência de sobrepeso abrange mais da metade da população brasileira $(55,7 \%)$ havendo diferença entre mulheres $20,7 \%$ e homens $18,7 \%$. Em comparação ao ano 2006, a obesidade no Brasil passou de 11,8\% para 19,8\%, entre 2006 e 2018, o aumento foi de 67\% (Brasil, 2018).

Diante disso, o manejo da etiologia é desafiador, visto que a obesidade é complexa e com muitos fatores envolvidos em seu desenvolvimento como dietas desequilibradas, estilo de vida sedentário, causas genéticas, emocionais e psicossociais. (WHO, 2020). De acordo com Silva Junior et al. (2017) atualmente são as principais causas de mortes no Brasil. É biologicamente considerada como um acúmulo de adipócitos nos tecidos, devido ao elevado índice de massa corporal (Bernhard et al., 2013).

Assim como também, a obesidade está diretamente relacionada com a ingestão de alimentos hipercalóricos e a diferentes composições da microbiota intestinal (Hartstra et al., 2015). Clarker et al. (2012) estabelece a obesidade a diversas patologias metabólicas como Diabetes Mellitus tipo 2, dislipidemias, a apneia ao sono, as doenças cardiovasculares e a alta mortalidade. Quanto maior é o excesso de peso, maior é a gravidade da doença. E uma das questões levantadas nos últimos anos relaciona a obesidade com a composição e funcionalidade da microbiota intestinal. Decerto, as ligações são evidências.

\subsection{Modulação Intestinal}

\subsubsection{Probióticos}

De acordo com a Organização Mundial de Saúde (OMS) probióticos são microrganismos vivos que quando consumidos em quantidades adequadas, conferem benefícios à saúde do hospedeiro. Ao longo da história da humanidade, microrganismos probióticos têm feito parte da alimentação humana na forma de produtos fermentados em todo mundo. A influência deste consumo em funções fisiológicas é cada vez mais documentada, com crescente interesse pelo seu possível efeito antiobesidade (Arora et al., 2012).

Os lactobacilos são bactérias gram-positivas e anaeróbicas facultativas, predominantes no intestino delgado. Entre suas espécies pode-se citar Lactobacillus casei, Lactobacillus rhamnosus, Lactobacillus acidophilus (OMGE, 2011). E também, os probióticos fazem parte dos chamados "Alimentos Funcionais", cujo principal alvo é a microbiota intestinal. Diferentes probióticos são capazes de induzir respostas pró-inflamatórias, anti-inflamatórias ou secretórias (Verspreet et al., 2016).

Certamente, diante das alterações metabólicas e das modificações da microbiota intestinal que ocorrem em indivíduos obesos, a utilização de probióticos na modulação dessas condições, surge como estratégia coadjuvante na prevenção 
e tratamento dessa condição clínica (Petschow et al., 2013). A Legislação Brasileira define que a quantidade mínima viável para um probiótico deve estar situada na faixa de 108 a 109 UFC (unidades formadoras de colônias) na porção diária do alimento para ser de importância fisiológica para o consumidor.

Outrossim, o consumo de quantidades adequadas dos microrganismos probióticos desejados nos bioprodutos (109 e 1010 UFC/100g de produto) é suficiente para a manutenção das concentrações ativas fisiologicamente (quantidade intestinal de 106 a 107 UFC/g) "in vivo" (Nogueira \& Gonçalves, 2011). Ainda assim, os probióticos auxiliam na produção de alguns compostos como vitaminas do complexo B, que são importantes para a proteção do fígado, influenciam no trânsito intestinal, ajudam na absorção de nutrientes, também podem aumentar o valor nutritivo dos alimentos e promove uma redução na acidez durante o armazenamento do produto final (Nogueira \& Gonçalves, 2011).

Ademais, a utilização de probióticos como modulação intestinal em indivíduos obesos é de fato satisfatório, posto que a obesidade é uma doença inflamatória mediada pelo estresse oxidativo e agentes inflamatórios que modificam a estrutura intestinal alterando bactérias boas e aumentando bactérias obeso gênicas como Basteroidetes e Firmicutes respectivamente. Dessa forma, a ação de microrganismos benéficos como probióticos das espécies Lactobacillus e Bifidobacterium seriam importantes para a diminuição dessas manifestações sistêmicas (Oliveira et al., 2017).

\subsubsection{Prebióticos}

Tão importante quanto os probióticos, são os prebióticos. Prebióticos são carboidratos não digeríveis que afetam e influenciam favoravelmente o hospedeiro, estimulando seletivamente a proliferação e funções de bactérias não patogênicas no cólon (Li, Wang \& Donavam, 2014). Melhor dizendo, são substâncias fermentadas que causam mudanças na formação e bemestar do hospedeiro. O termo prebiótico é utilizado para determinados componentes de alimentos de origem vegetal que não são digeríveis em nenhuma das etapas do processo digestório e assim resistentes a ação de enzimas (Ludimilla \& Fabiola, 2015).

O uso de prebióticos traz inúmeros benefícios, pois estimulam o crescimento e atividade de uma ou mais espécies do cólon. Já se sabe que alguns nutrientes possuem propriedades funcionais e os prebióticos são exemplos desses alimentos, por proporcionar em efeitos benéficos no organismo especialmente melhorias na microbiota intestinal, e do cólon que é de extrema importância para o equilíbrio da mesma, para saúde e manutenção (Gritz \& Bhandari, 2015). O autor ainda refere que a composição é por carboidratos de distintos tamanhos como monossacarídeos, oligossacarídeos e até polissacarídeos.

Segundo Raizel et al. (2011) os prebióticos são encontrados em alimentos como beterraba, banana, alho, cebola, entre outros. E para que se obtenham efeitos, é necessário o consumo diário de 18 a 20 gramas. Além do mais, o êxito dos prebióticos depende essencialmente da sua não hidrolisação pelas enzimas digestivas, possibilitando assim, atingir o intestino grosso intacto, lugar que ocorrerá a sua fermentação e digestão. Sua função principal é estimular o crescimento e/ou ativar o metabolismo de bactérias não patogênicas no TGI. Atuam, por sua vez, bloqueando sítios de aderência, imobilizando e reduzindo a capacidade de fixação de algumas bactérias patogênicas no intestino (Brito et al., 2014).

Portanto, os probióticos e prebióticos agem diretamente interligados e essa simetria gera os produtos simbióticos. O consumo desses nutrientes eleva a ação benéfica de cada um deles, devido ao estímulo de cepas probióticas conhecidas que levam à escolha de pares simbióticos substrato-microrganismo ideais para o TGI (Raizel et al., 2011).

\subsection{Influências na saúde intestinal}

\subsubsection{Dieta Ocidental}

O comportamento alimentar está diretamente ligado ao estilo e hábitos de vida do indivíduo. O hábito de vida ocidental conduz aos hábitos inadequados que constituem em doenças e agravos não transmissíveis como obesidade, 
hipertensão arterial e diabetes mellitus. Os maus costumes e a falta de cuidado com a função intestinal fazem com que as bactérias negativas aumentem, causando um desequilíbrio da microbiota intestinal (Galdino et al., 2016).

Em virtude disso, a alimentação pode ser considerada um dos fatores que mais influenciam a qualidade de vida dos indivíduos (Ferreira, 2014). Além disso, as alterações da permeabilidade intestinal e a disbiose intestinal podem ocorrer devido interações entre nutrientes decorrentes de uma má absorção, resultante de uma alimentação inadequada (Amarante, 2013).

Pesquisa de Jumpertz et al. (2011) mostrou que a disponibilidade de diferentes nutrientes provoca mudanças na composição da microbiota intestinal no mecanismo de absorção dos nutrientes. Nesse sentido, a microbiota tanto de humanos como de animais pode ser modificada através da dieta, de modo que a ingestão de calorias em excesso promove a proliferação de bactérias do filo firmicutes. Não somente, o autor também mostrou que em apenas 3 dias a estrutura da microbiota intestinal humana pode ser drasticamente alterada por variações na ingestão de calorias (2.400 kcal - 3.400 kcal diárias).

Por outro lado, a qualidade da dieta tem se mostrado um importante modulador da composição da microbiota intestinal, no que diz respeito ao teor de gordura ingerido por determinado indivíduo. A ingestão de gordura em excesso afeta de maneira direta a integridade da mucosa intestinal e assim prejudica a sua permeabilidade (Moraes, 2016). Por fim, os hábitos alimentares ocidentais têm sido responsáveis pelo aumento de doenças inflamatórias por se tratar de uma dieta rica em calorias e gorduras saturadas e pobre em nutrientes como fibras e o ômega-3 (Vieira, 2011).

\subsubsection{Disbiose Intestinal}

O intestino é um órgão importante do nosso sistema imunológico, pois uma microbiota saudável auxilia na digestão e absorção de nutrientes diminuindo os riscos de algumas patologias. Uma microbiota em desequilíbrio faz com que as bactérias negativas se alojem no intestino delgado, causando sérios danos, como por exemplo má absorção de nutrientes e formação de toxinas (Ferreira, 2014). Ou seja, a função adequada da microbiota intestinal depende de uma composição celular estável, que, no caso da microbiota humana, consiste principalmente de bactérias dos filos Firmicutes, Bacteroidetes e Actinobactéria. Assim, mudanças nas razões desses filos ou expansão de novos grupos bacterianos levam a um desequilíbrio, ocasionando a disbiose (Weiss \& Hennet, 2017).

A Disbiose trata-se de uma desordem na microbiota intestinal, ocasionada por um desajuste da colonização bacteriana, onde bactérias nocivas estão em maior quantidade que bactérias benéficas. Dentre os causadores da disbiose intestinal destacamos o uso irregular de fármacos, principalmente o uso de antibióticos destruindo as bactérias benéficas e favorecendo o crescimento de fungos que causam irritação da mucosa intestinal (Ferreira, 2014). Outrossim, diversos fatores foram relacionados ao desenvolvimento da disbiose intestinal como tipos de parto, aleitamento, deficiência na produção de ácido clorídrico, dieta deficiente, abuso de medicamentos, estresse, idade e baixa imunidade (Bronw et al., 2012).

De acordo com Gagliardi et al. (2018) a disbiose intestinal também pode estar associada a intolerâncias alimentares decorrentes da deficiência de enzimas digestivas, por exemplo, a deficiência de lactose, que promove intolerância ao leite. Isto é, são vários os fatores que influenciam no desencadeamento da disbiose. A sua relação com a obesidade é evidente, visto que certas bactérias e fungos que habitam o intestino humano são capazes de reabsorver amido e açúcar no colón (Kercher \& Garcia 2016).

O tratamento da disbiose intestinal pode ser realizado com a utilização de medicamentos e através da mudança de hábitos alimentares. Recomenda-se, portanto, o consumo de alimentos orgânicos, livres de agrotóxicos e de aditivos, isenta de industrializados e de caráter irritativo (Vieira, 2016). Dentro desse contexto, de Souza e Fernandes (2015), fizeram referência na importância da utilização de simbióticos (prebióticos e probióticos) como modulação intestinal.

Dos Santos Moraes et al. (2018) comenta sobre o Kefir, bebida composta de leite fermentado associado a leveduras e bactérias, como alternativa no tratamento de pacientes com disbiose intestinal, já que contribui para a redução nos níveis 
séricos de glicose e colesterol LDL. Além disso, outra opção vem sendo estudada no tratamento de disbiose, como o Kombucha, por possuir compostos bioativos semelhantes ao chá verde. Kombucha é uma bebida fermentada de chá verde e/ou chá preto adoçados, no qual é adicionado uma cultura contendo um consórcio simbiótico de bactérias e leveduras (Paludo, 2017).

Ainda dentro do contexto, a banana é reconhecida como um alimento potencialmente benéfico no tratamento da disbiose, especificamente a verde cozida, pois apresenta amido resistente, o qual está associado a diversos benefícios para a saúde intestinal (Gomes et al., 2017). E um dos seus derivados, a biomassa de banana verde, auxilia no controle de glicemia, colesterol e ajuda no tratamento de distúrbios intestinais. Acredita-se que esse alimento promova benefícios na microbiota intestinal, modificando a microflora do cólon e aumentando a excreção fecal de nitrogênio. Tal ação, reduz de maneira importante o câncer de cólon (Gomes et al., 2017).

Não só, para que o tratamento da disbiose obtenha sucesso, é indicado retirar alimentos alergênicos como glúten, leite e derivados, xenobióticos e ainda excesso de alimentos industrializados. Em seguida, introduzir colônias de probióticos e dieta balanceada, não irritativa, rica em nutrientes protetores (De Souza \& Fernantes, 2015). Dessa forma, sabe-se que a disbiose intestinal é uma condição favorável para diversas doenças crônicas, como por exemplo a obesidade.

\subsubsection{Oligossacarídeos, Dissacarídeos, Monossacarídeos e Polióis fermentáveis (FODMAP's)}

FODMAP's são hidratos de carbono de acordo com seu comprimento, e resultou na sigla com significado (Oligossacarídeos, Dissacarídeos, Monossacarídeos e Polióis Fermentáveis) (Barret \& Gibson, 2012). Os FODMAP's têm propriedades funcionais comuns. São mal absorvidos no intestino delgado e são rapidamente fermentados por bactérias: a rapidez de fermentação é ditado pelo comprimento da cadeia de hidratos de carbono (quanto mais pequenas, mais rapidamente fermentáveis) (El-Salhy \& Gundersen, 2015; Pedersen, 2014).

Há dois conceitos-chaves importantes sobre os FODMAP's: o primeiro diz respeito à abordagem dietética com restrição desses hidratos de carbono globalmente. O segundo, diz respeito à não participação destes hidratos de carbono de cadeia curta como causa da doença funcional, mas sim, como possibilidade de reduzir os sintomas associados a esta condição, quando se eliminam da dieta (Gibson \& Shepherd, 2010).

Certamente, a dieta baixa em FODMAP's auxilia pacientes a controlar sintomas gastrointestinais e posteriormente identificar os desencadeadores alimentares específicos. O período é determinado, não sendo recomendada a dieta por longos períodos (Yao et al., 2014). Alimentos ricos em FODMAP's, por exemplo, a serem evitados, são maçã, damasco, manga, pera, melancia, alcachofra, cebola, couve-flor, castanha de caju, trigo, leites e derivados, mel, sorbitol, frutose, entre outros (Barrett, 2013). Logo, o que se come define em grande medida a microbiota gastrointestinal (Payne, Chassard \& Lacroix, 2012).

Além do mais, quando ingeridos os FODMAPs aumentam a carga osmótica ao nível do lúmen intestinal, particularmente no intestino delgado, o que se traduz em distensão, e paralelamente, por ação das bactérias do cólon dá-se a produção de gases, como o dióxido de carbono, metano e hidrogênio (Spiller, 2017). Em indivíduos mais suscetíveis, esta estimulação local pode manifestar-se através de dor, distensão abdominal, flatulência e alteração da motilidade intestinal, nomeadamente diarreia (Hill, Muir \& Gibson, 2017).

Somado a isso, uma dieta baixa em FODMAPs pode melhorar ou piorar a qualidade de vida do indivíduo; porém, a maioria dos casos sugere que a dieta baixa em FODMAPs não produz uma deterioração na qualidade de vida e ela pode até melhorá-la (Ostgaard et al., 2012). Dessa forma, embora haja diferenças na magnitude de como cada tipo de FODMAP atinge o trato gastrointestinal inferior, coletivamente, eles foram atribuídos ao aumento dos sintomas gastrointestinais funcionais em indivíduos suscetíveis na SII, e sua exclusão alimentar como grupo tem uma maior magnitude na melhora dos sintomas do que cada um isoladamente (Lomer, 2014). 


\section{Conclusão}

O desenvolvimento do presente estudo possibilitou explanar a compreensão da importância em manter um equilíbrio na microbiota intestinal, visto que sua desordem pode ser uma das causas da obesidade no ser humano. Sabe-se que através dessa estabilidade entre as comunidades microbianas, pode-se garantir uma condição clínica saudável auxiliando no bom prognóstico, evitando intolerâncias alimentares, disbiose intestinal e auxiliando na modulação de respostas inflamatórias.

O estudo também permitiu elucidar a notoriedade no tratamento da microbiota intestinal com simbióticos, probióticos e prebióticos, além da melhoria na qualidade dos hábitos alimentares ocidentais, restrição dos FOODMAP's, entre outros. Sendo assim, é possível consentir a relevância em manter uma microbiota intestinal saudável e equilibrada a fim de evitar complicações proeminentes da obesidade e reconhecer que a modulação intestinal é imprescindível no tratamento do paciente obeso.

\section{Referências}

Amarante, D. (2013). Aspectos nutricionais na população de pacientes com síndrome do intestino irritável atendidos no Hospital das Clínicas da Faculdade de Medicina da Universidade de São Paulo. 93f. Dissertação (Mestrado em Ciências em Gastroenterologia) - Faculdade de Medicina do estado de São Paulo, São Paulo. https://www.teses.usp.br/teses/disponiveis/5/5168/tde-09082013-120311/publico/DaianaAmarante.pdf.

Arora, T., Anastasovska, J., Gibson, G., Tuohy, K., Sharma, R., \& Bell, J. (2012). Effect of Lactobacillus acidophilus NCDC 13 supplementations on the progression of obesity in diet- induced obese mice. Br J Nutr, [S.1.], 108, 1382-1389.

Barbosa, F. et al. (2010). Microbiota indígena do trato gastrintestinal. Revista de Biologia e Ciência da Terra, Aracaju, 10(1), 78-93.

Barrett, J. S. (2013). Extending our knowledge of fermentable, short-chain carbohydrates for managing gastrointestinal symptoms. Nutr Clin Pract, [S.1.], 28(3), 300-6.

Barrett, J. S., \& Gibson, P. R. (2012). Fermentable oligosaccharides, disaccharides, monosaccharides and polyols (FODMAPs) and nonallergic food intolerance: FODMAPs or food chemicals? Therapeutic Advances in Gastroenterology, 5(4), 261-268.

Bello, D., Laboy, D. J., Shen, N., Cox, L. M., González, A., Bokulich, N. A., Canção, S. J., Hoashi, M., Rivera, V. J. I., Mendez, K., Cavaleiro, R., \& Clemente, J. C. (2016). Partial restoration of the microbiota of children born by cesarean section via vaginal microbial transfer. Nat Med, 22(3). 250-3.

Bernhard, F. et al. (2013). Functional relevance of genes implicated by obesity genome-wide association study signals for human adipocyte biology. Diabetologia, 56, 311.

Bervoets, L. et al. (2013). Differences in gut microbiota composition between obese and lean children: a cross-sectional study. Gut Pathogens, 5(1)1, 10.

Brasil. (2018). Ministério da Saúde. VIGITEL BRASIL 2017: vigilância de fatores de risco e proteção para doenças crônicas por inquérito telefônico. Brasília: Ministério da Saúde. https://sbpt.org.br/portal/wp-content/uploads/2019/01/VIGITEL-2017-18.pdf.

Brito, J. et al. (2014). Probiótico, prebiótico e simbiótico na alimentação de não-ruminantes. Revista Eletrônica Nutrime, Viçosa, 11(1), 3070-3084.

Brown, K. et al. (2012). Diet: Induced Dysbiosis of the intestinal microbiota and the effects on immunity and disease. Nutrients, [S.1.], 4(8), 1095-1119.

Clarker, S. F., \& Murphy, E. F. et al. (2012). The gut microbiota and its relationship to diet and obesity: New insights. Gut Microbes, [S.1.], 3(3), 186-202.

De Souza, M. V. O., \& Fernandes, L. A. B. (2015). Nutrição funcional aplicada na disbiose intestinal. In: Congresso de iniciação científica-UNIFEV, Votuporanga. Anais... UNIFEV, Votuporanga. 397-398.

El-salhy, M., \& Gundersen, D. (2015). Diet in irritable bowel syndrome. Nutrition Journal, [S.1.], 14, 2-11.

FAO. WHO. (2001). Health and Nutritional Properties of Probiotics in Food including Powder Milk with Live Lactic Acid Bacteria. ftp://ftp.fao.org/docrep/fao/009/a0512e/a0512e00.pdf.

Ferreira, G. S. (2014). Disbiose intestinal: Aplicabilidade do probiótico e dos prebióticos na recuperação e manutenção da microbiota intestinal. 33f. Monografia (Graduação em Farmácia) - Centro Universitário Luterano de Palmas, Palmas-TO. https://ulbrato.br/bibliotecadigital/uploads/document55e9f4b59e0bd.pdf.

Gagliardi, A. et al. (2018). Rebuilding the Gut Microbiota Ecosystem. Int J Environ Res Public Health, 15(8).

Galdino, J. J., Oselame, G. B., Oselame, C. S., \& Neves, E. B. (2016). Questionário de rastreamento metabólico voltado a disbiose intestinal em profissionais de enfermagem. RBONE - Revista Brasileira de Obesidade, Nutrição e Emagrecimento, [S.1.], 10(57), 117-122, http://www.rbone.com.br/index.php/rbone/article/view/422.

Gibson, P. R., \& Shepherd, S. J. (2010). Evidence-based dietary management of functional gastrointestinal symptoms: The FODMAP approach. J Gastroenterol Hepatol, [S.1.], 25(2), 252-258. https://pubmed.ncbi.nlm.nih.gov/20136989/.

Gomes, V. T. S. et al. (2017). Benefícios da biomassa de banana verde à saúde humana. Revista Univap, 22(40), 655. 
Gritz, E. C., \& Bhandari, V. (2015). The human neonatal gut microbiome: a brief review. Front Pediatr, [S.1.], 3(17). https://pubmed.ncbi.nlm.nih.gov/25798435/.

Hartstra, A. V., Bouter, K. E. C., Bäckhed, F., \& Nieuwdorp, M. (2015). Insights into the Role of the Microbiome in Obesity and Type 2 Diabetes. Diabetes Care, [S.1.], 38(1), 159-165.

Hill, C. et al. (2014). The international scientific association for probiotics and prebiotics consensus statement on the scope and appropriate use of the term probiotic. Nature Reviews Gastroenterology \& Hepatology, [S.1.], 11, 506-514.

Hill, P.; Muir, J. G., \& Gibson, P. R. (2017). Controversies and Recent Developments of Low-FODMAP Diet. Gastroenterology \& Hepatology, [S.1.], 13(1).

Jumpertz, R., Le, D. S., Turnbaugh, P. J., Trinidad, C.; Bogardus, C., Gordon, J. I., \& Krakoff, J. (2011). Energy-balance studies reveal associations between gut microbes, caloric load, and nutrient absorption in humans. American Journal of Clinical Nutrition, Bethesda, 94(1), 58-65.

Kercher, K. K. O., \& Garcia, M. C. R. (2016). Correlação da disbiose intestinal e obesidade: uma revisão bibliográfica. In: seminário de iniciação científica, 24, Rio Grande do Sul. 1-4.

Li, M.; Wang, M., \& Donavan, S. M. (2014). Early development of the gut icrobiome and immune-mediated childhood disorders. Seminars in Reproductive Medicine, New York, 32(1), 74-86.

Lomer, M. C. E. (2014). Review article: the aetiology, diagnosis, mechanisms and clinical evidence for food intolerance. Alimentary Pharmacology \& Therapeutics, [S.1.], 41(3), 262-275.

Ludimilla, A. P., \& Fabiola, F. S. C. (2015). Colonização da microbiota intestinal e sua influência na saúde do hospedeiro. Universitas: Ciências da Saúde, 14(1), 85-96. https://www.publicacoesacademicas.uniceub.br/cienciasaude/article/view/3629.

Matamoros, S., Gras-Leguen, C., Le Vascon, F., Potel, G., \& De La Cochetiere, M. F. (2013). Development of intestinal microbiota in infants and its impact on health. Trends Microbiol, [S.1.], 21(4), 167-73. https://pubmed.ncbi.nlm.nih.gov/23332725/.

Million, M. et al. (2013). Correlation between body mass index and gut concentrations of Lactobacillus reuteri, Bifidobacterium animalis, Methanobrevibacter smithii and Escherichia coli. International journal of obesity, [S.1.], 37(11), 1460-1466.

Moraes, F. C. A. (2016). Análise da Microbiota intestinal em adultos com hábitos alimentares distintos e de associação com a inflamação e resistência à insulina. 115f. Tese (Doutorado em Nutrição em Saúde Pública) - Faculdade de Saúde Pública, USP, São Paulo. https://www.teses.usp.br/teses/disponiveis/6/6138/tde-03052016-144159/publico/AnaCarolinaFrancoDeMoraesREVISADArestrita.pdf.

Murphy, E. F. et al. (2010). Composition and energy harvesting capacity of the gut microbiota: relationship to diet, obesity and time in mouse models. Gut, [S.1.], 59(12), 1635-42. https://pubmed.ncbi.nlm.nih.gov/20926643/.

Nogueira, J. C. R.; Gonçalves, R. C. M. (2011). Probióticos - Revisão da Literatura. Revista Brasileira de Ciências da Saúde, [S.1.], 15(4), 487-92.

Oliveira, J. M. S., Santos, G. M., Saldanha, N. M. V. P., Sousa, P. V. L., \& Carvalho, A. C. S. (2017). Efeitos de probióticos no tratamento da obesidade. Revista Eletrônica da FAINOR, Vitória da Conquista, 10(21), 154-165.

OMGE. Organização mundial de gastroenterologia. (2011). Guias Mundiais da WGO: Probióticos e Prebióticos. https://www.worldgastroenterology.org/UserFiles/file/guidelines/probiotics-portuguese-2011.pdf.

Ostgaard, H., Hausken, T., Gundersen, D., \& El-Salhy, M. (2012). Diet and effects of diet management on quality of life and symptoms in patients with irritable bowel syndrome. Mol Med Rep, [S.1.], 5(6), 1382-90.

Paludo, N. (2017). Desenvolvimento e caracterização de kombucha obtida a partir de chá verde e extrato de erva-mate: processo artesanal e escala laboratorial. (Monografia)- Universidade Federal do Rio Grande do Sul, Instituto de Ciência e Tecnologia de Alimentos, Porto Alegre.

Park, J. S., Seo, J. H., \& Youn, H. S. (2013). Gut microbiota and clinical disease: obesity and nonalcoholic fatty liver disease. Pedriatr Gastroenterol Hepatol Nutri, [S.1.], 16(1), 22-7.

Payne, A. N., Chassard, C., \& Lacroix, C. (2012). Gut microbial adaptation to dietary consumption of fructose, artificial sweeteners and sugar alcohols: implications for host-microbe interactions contributing to obesity. Obes Rev, [S.1.], 13(9), 799-809.

Pedersen, N. (2014). Ehealth monitoring in irritable bowel syndrome patients treated with low fermentable oligo-, di-, mono-saccharides and polyols diet. World Journal of Gastroenterology, [S.1.], 20, 21.

Perovano, D. G. (2014). Manual de Metodologia Científica. Paraná: Editora Juruá.

Petschow, B., Doré, J., Hibberd, P., Dinan, T., Reid, G., Blaser, M. et al. (2013). Probiotics, prebiotics, and the host microbiome: the science of translation. Ann N Y Acad Sci, [S.1.], 1306(1), 1-17.

Raizel, R., Santini, E.; Kopper, A. M., \& Reis Filho, A. D. (2011). Efeitos de probióticos, prebióticos e simbiótico para o organismo humano. Revista Ciência \& Saúde, [S.1.], 4(2). https://revistaseletronicas.pucrs.br/ojs/index.php/faenfi/article/view/8352.

Rodrigues, A. (2011). Microbiota intestinal e sua possível relação com a obesidade. ABESO, 53(5), 5-7.

Santos, K. E. R., \& Ricci, G. C. L. (2016). Microbiota intestinal e a obesidade. Revista Uningá Review, Maringá, 26(1), 74-82.

Santos Moraes, M. et al. (2018). Efeitos funcionais dos probióticos com ênfase na atuação do kefir no tratamento da disbiose intestinal. UNILUS Ensino e Pesquisa, [S.1.], 14(37), 144-156. 
Research, Society and Development, v. 10, n. 6, e52110616086, 2021

(CC BY 4.0) | ISSN 2525-3409 | DOI: http://dx.doi.org/10.33448/rsd-v10i6.16086

Santos, R., \& Varavalho, M. (2011). A importância de probiótico para o controle e/ou reestruturação da microbiota intestinal. Revista Científica do ITPAC, São Paulo, 4(1), 40-49.

Silva Junior, V. L., Lopes, F. A. M., Albano, R. M., Souza, M. G. C., Barbosa, C. M. L., Maranhão, P. A. et al. (2017). Obesity and gut microbiota: what do we know so far? Medical Express, [S.1.], 4(1), 1-11.

Silva, S. T., Santos, C. A., Bressan, J. (2013). Intestinal microbiota; relevance to obesity and modulation by prebiotics and probiotics. Nutr Hosp, 28(4), 103948.

Spiller, R. (2017). How do FODMAPs work? J Gastroenterol Hepatol, [S.1.], 32(1), 36-39.

Stenman, Lotta K. et al. (2015). Establishing a causal link between gut microbes, body weight gain and glucose metabolism in humans-towards treatment with probiotics. Beneficial Microbes, [S.1.], 7(1), 11-22.

Tremaroli, V., \& Bäcked, F. (2012). Functional interactions between the gut microbiota and host metabolim. Nature, 489(7415), 242-9.

Valero, Y., Colina, J., \& Herrera, H. (2015). La microbiota intestinal y su rol en la diabetes. Anales Venezolanos de Nutrición: Venezuela, Venezuela, 28(2), $132-144$.

Verspreet, J., Damen, B., Broekaert, W. F., Verbeke, K., Delcour, J. A., \& Courtin, C. M. (2016). A critical look at prebiotics within the dietary fiber concept. Аппи Rev Food Sci Technol, [S.1.], 7, 167-90.

Vieira T. A. (2011). Microbiota, dieta e sistema imune: um diálogo constante via ativação do receptor acoplado à proteína-G 43 (Gpr43). $69 \mathrm{f}$. Tese (Doutorado em Imunologia) - Universidade Federal de Minas Gerais, Belo Horizonte, MG. https://repositorio.ufmg.br/handle/1843/BUOS-9GYKEY.

Vieira, C. R. (2016). Efeito da abordagem nutricional e o uso de probióticos no tratamento da disbiose. 28f. Monografia (Especialização em Nutrição Clínica Funcional) - Universidade Cruzeiro do Sul, São Paulo. https://www.vponline.com.br/portal/noticia/pdf/af9d0df2dc55dda11238d3104ef6eae2.pdf.

Weiss, G. A., \& Hennet, T. (2017). Mechanisms and consequences of intestinal dysbiosis. Cell Mol Life Sci, [S.1.], 74(16), 2959-77.

WHO. (2020) Obesity and overweight. Fact sheet no. 311. Who media center. http://www.who.int/mediacentre/factsheets/fs311/en/.

Wu, G. D., Chen, J., Hoffmann, C., Bittinger, K., Chen, Y., Sue, A. et al. (2011). Linking long-term dietary patterns with gut microbial enterotypes. Science, [S.1.], 334(6052), 105-8.

Yao, C. K. et al. (2014). Dietary sorbitol and mannitol: food content and distinct absorption patterns between healthy individuals and patients with irritable bowel syndrome. J Hum Nutr Diet, [S.1.], 27(2), 263-75. 